\title{
Identificação das Regiões de Saúde do Rio Grande do Sul, Brasil, prioritárias para ações de Vigilância em Saúde
}

\author{
Identification of priority Health Regions in the State of Rio Grande \\ do Sul, Brazil, for Health Surveillance actions
}

Renata Petzhold Mondini ${ }^{1}$

Ivone Andreatta Menegolla ${ }^{1}$

Eduardo Viegas da Silva ${ }^{1}$

${ }^{1}$ Centro Estadual de Vigilância em Saúde, Secretaria de Estado da Saúde do Rio Grande do Sul. R. Domingos Crescencio 132/305, Santana. 90050-900 Porto Alegre RS Brasil. renata.mondini@ yahoo.com.br
Abstract This paper aimed to identify the priority Health Regions of Rio Grande do Sul (RS) to implement Health Surveillance strengthening actions. This is a descriptive study with data from time series of 11 (eleven) Health Surveillance indicators of the Ministry of Health's 2016 Guidelines, Objectives, Targets and Indicators Journal agreed by the Bipartite Interagency Committeel RS. The selected indicators are synthesized to produce a Composite Health Surveillance Assessment Indicator (ICAVES) for each of the 30 Health Regions of the state, creating values ranging from zero (worst) to 1 (best), using the Human Development Index (HDI) construction calculation method. The lowest rates of the composite indicator are found in the Health Regions "20-Rota da Produção" and "19-Região do Botucaraí". These two Health Regions are priorities for the strengthening of collective Health Surveillance actions and the management of health risks and diseases, considering horizontal equity as guideline of the Unified Health System.

Key words Public Health Surveillance, Regional health planning, Health status indicators, Health priorities, Health equity
Resumo O presente artigo identifica as Regiões de Saúde do Rio Grande do Sul (RS) prioritárias com vistas a implementar ações para o fortalecimento da Vigilância em Saúde. Estudo descritivo com dados da série histórica de 11 (onze) indicadores de Vigilância em Saúde do Caderno de Diretrizes, Objetivos, Metas e Indicadores 2016, do Ministério da Saúde pactuados pela Comissão Intergestores Bipartite/RS. Os indicadores selecionados são sintetizados para produzir um Indicador Composto de Avaliação da Vigilância em Saúde (ICAVES) para cada uma das 30 Regiões de Saúde do estado, criando valores que variam de 0 (pior) a 1 (melhor), tendo como método de cálculo o utilizado para a construção do Índice de Desenvolvimento Humano (IDH). Os menores indices do indicador composto estão nas Regiões de Saúde: 20-Rota da Produção e 19-Região do Botucaraí. As Regiões de Saúde 20 e 19 são prioritárias para o fortalecimento das ações de âmbito coletivo da Vigilância em Saúde e o gerenciamento de riscos e agravos à saúde considerando a equidade horizontal como diretriz do Sistema Único de Saúde. Palavras-chave Vigilância em Saúde, Regionalização, Indicadores em saúde, Prioridades em saúde, Equidade em saúde 


\section{Introdução}

No Rio Grande do Sul, o processo de regionalização do Sistema Único de Saúde (SUS) instituiu 30 (trinta) Regiões de Saúde em 2012 com a finalidade de integrar a organização, o planejamento e a execução das ações e dos serviços de saúde ${ }^{1}$. A partir disso, o planejamento da gestão no Rio Grande do Sul é baseado na organização do território por Regiões de Saúde ${ }^{1-3}$.

O Caderno de Diretrizes, Objetivos, Metas e Indicadores 2016 do Ministério da Saúde (MS) é um dos instrumentos de gestão do SUS utilizados para planejamento em saúde, tendo como objetivo orientar o processo nacional de pactuação interfederativa. O documento apresenta as fichas de qualificação de 29 indicadores de saúde estabelecidos para o ano de 2016, divididos em universais de pactuação comum e obrigatória, e específicos, de pactuação obrigatória conforme especificidades no território ${ }^{4}$.

O Ministério da Saúde para cada um dos 29 indicadores recomenda parâmetros nacionais de referência para a pactuação das metas. Entre estes, no ano de 2016, a Comissão Intergestora Bipartite do Rio Grande do Sul (CIB/RS) pactuou 11 (onze) indicadores da Vigilância em Saúde ${ }^{5}$. Assim, os técnicos do Centro Estadual de Vigilância em Saúde (CEVS), para subsidiar a pactuação, disponibilizaram a série histórica dos 11 indicadores, sendo que 5 (cinco) tiveram suas metas pactuadas com valores abaixo da referência recomendada pelo MS e 1 (um), com valor acima ${ }^{5,6}$.

Os indicadores de saúde vistos em conjunto e regularmente num sistema dinâmico proporcionam fundamentos para avaliação epidemiológica da situação da saúde e, consequentemente, para as intervenções prioritárias na realidade sanitária dos territórios? ${ }^{7}$.

Tendo como base o cálculo do Índice de Desenvolvimento Humano (IDH), Silva Junior ${ }^{8}$ construiu um Indicador Composto para Avaliação do Desempenho da Vigilância em Saúde (ICAVES), o qual sintetiza em um único valor um dado rol de indicadores.

No contexto da heterogeneidade da organização do sistema de saúde entre as regiões, o presente estudo tem como objetivo avaliar o desempenho da Vigilância em Saúde no Rio Grande do Sul e em suas Regiões de Saúde, a partir dos 11 (onze) indicadores da Vigilância em Saúde pactuados na CIB/RS para o ano de 2016. Com isso, busca identificar as Regiões de Saúde mais vulneráveis que requerem maior apoio institucional, visando promover equidade em saúde ${ }^{3,9-11}$.

\section{Métodos}

Estudo de natureza aplicada, descritivo e de abordagem quantitativa, realizado com dados secundários disponibilizados no site da Secretaria Estadual de Saúde do Rio Grande do Sul ${ }^{6,12}$.

O trabalho foi construído com a série histórica, por Região de Saúde, de 11 (onze) indicadores da Vigilância em Saúde constantes no Caderno de Diretrizes, Objetivos, Metas e Indicadores/ MS/2016 pactuados pela Comissão Intergestores Bipartite/RS para o ano de 2016,5.5. Estão compreendidos nesse rol indicadores de vigilância: ambiental, epidemiológica, sanitária e de saúde do trabalhador.

O período da série histórica compreende 7 anos (2009-2015), contudo 4 indicadores possuem uma série histórica menor. Para seleção dos indicadores foram utilizados três critérios: constarem no Caderno de Diretrizes, Objetivos, Metas e Indicadores/MS/2016; serem pactuados pela Comissão Intergestores Bipartite/RS para o ano de 2016; e serem de Vigilância em Saúde.

No Quadro 1 são apresentados os indicadores selecionados, a vigilância a que pertencem, as fontes de dados e os períodos utilizados para o cálculo da série histórica.

A partir da seleção dos indicadores se construiu um Indicador Composto de Avaliação da Vigilância em Saúde (ICAVES) que sintetiza em um único valor - Índice Parcial - o desempenho da Vigilância em Saúde para o estado do Rio Grande de Sul e para suas 30 Regiões de Saúde ${ }^{6}$.

A metodologia para construção do ICAVES, descrita na literatura, é baseada no cálculo do Îndice de Desenvolvimento Humano (IDH), visto que é um indicador composto amplamente utilizado que sintetiza os índices parciais de longevidade, educação e renda, que variam entre os valores 0 (pior) e 1 (melhor), em um único ${ }^{6}$.

Inicialmente, para construir o ICAVES foi necessário estabelecer parâmetros, conforme a realidade estadual, para os valores das variáveis utilizadas na fórmula de cálculo do Índice Parcial, a saber: valor observado, valor mínimo e valor máximo. O índice Parcial é a razão entre o valor observado menos o valor mínimo e valor máximo menos o valor mínimo.

Na variável valor observado foi utilizada a média da série histórica de cada indicador para cada uma das 30 Regiões de Saúde. Logo, cada Região de Saúde possui 11 médias - perfazendo um total de 330 (30 Regiões de Saúde x 11 Indicadores).

O valor atribuído para a variável valor mínimo é a média da série histórica da Região de 
Quadro 1. Seleção de indicadores da Vigilância em Saúde pactuados em CIB/RS, fonte de dados utilizada para cálculo da série histórica.

\begin{tabular}{|c|c|c|c|c|}
\hline \multirow{2}{*}{ Vigilância } & \multirow{2}{*}{ Indicador } & \multirow{2}{*}{ Fonte } & \multicolumn{2}{|c|}{ Série Histórica } \\
\hline & & & Período & Anos \\
\hline Epidemiológica & $\begin{array}{l}\text { Taxa de mortalidade prematura (de } 30 \text { a } 69 \text { anos) pelo } \\
\text { conjunto das quatro principais doenças crônicas não } \\
\text { transmissíveis (DCNT - doenças do aparelho circulatório, } \\
\text { câncer, diabetes e doenças respiratórias crônicas) }\end{array}$ & SIM & $2010-2015$ & 6 \\
\hline Epidemiológica & $\begin{array}{l}\text { Proporção de vacinas do Calendário Básico de Vacinação da } \\
\text { Criança com coberturas vacinais alcançadas }\end{array}$ & SIPNI & 2009-2015 & 7 \\
\hline Epidemiológica & $\begin{array}{l}\text { Proporção de cura de casos novos de tuberculose pulmonar } \\
\text { com confirmação laboratorial }\end{array}$ & SINAN & $2009-2015$ & 7 \\
\hline Epidemiológica & $\begin{array}{l}\text { Proporção de exame anti-HIV realizados entre os casos } \\
\text { novos de tuberculose }\end{array}$ & SINAN & $2009-2015$ & 7 \\
\hline $\begin{array}{l}\text { Saúde do } \\
\text { Trabalhador }\end{array}$ & $\begin{array}{l}\text { Proporção de municípios com casos de doenças ou agravos } \\
\text { relacionados ao trabalho notificados }\end{array}$ & SINAN & 2011-2015 & 5 \\
\hline Epidemiológia & $\begin{array}{l}\text { Proporção de cura dos casos novos de hanseníase } \\
\text { diagnosticados nos anos das coortes }\end{array}$ & SINAN & 2009-2015 & 7 \\
\hline Epidemiológica & $\begin{array}{l}\text { Proporção de contatos examinados de casos novos de } \\
\text { hanseníase }\end{array}$ & SINAN & $2009-2015$ & 7 \\
\hline Epidemiológica & Número absoluto de óbitos por dengue & SINAN & $2009-2015$ & 7 \\
\hline Ambiental & $\begin{array}{l}\text { Proporção de imóveis visitados em, pelo menos, quatro } \\
\text { ciclos de visitas domiciliares para controle da dengue }\end{array}$ & SISPNCD & $2014-2015$ & 2 \\
\hline Ambiental & $\begin{array}{l}\text { Proporção de análises realizadas em amostras de água para } \\
\text { consumo humano quanto aos parâmetros coliformes totais, } \\
\text { cloro residual livre e turbidez }\end{array}$ & $\begin{array}{l}\text { SISAGUA/ } \\
\text { MS }\end{array}$ & $2009-2015$ & 7 \\
\hline Sanitária & $\begin{array}{l}\text { Percentual de municípios que realizam no mínimo seis } \\
\text { grupos de ações de Vigilância Sanitária, consideradas } \\
\text { necessárias a todos os municípios }\end{array}$ & SIA/SUS & $2012-2015$ & 4 \\
\hline
\end{tabular}

Siglas: SIM - Sistema de Informação de Mortalidade; SIPNI - Sistema de Informação do Programa Nacional de Imunizações; SINAM - Sistema de Informação de Agravos de Notificação; SISPNCD - Sistema do Programa Nacional de Controle da Dengue; SIA/SUS - Sistema de Informações Ambulatoriais do SUS.

Saúde com menor desempenho em cada um dos indicadores.

Quando à variável valor máximo, foram adotados três parâmetros: (1) Meta recomendada pelo Ministério da Saúde para pactuação do indicador no ano de 2016, (2) Meta pactuada na CIB/ RS para o indicador no ano de 2016 e (3) média da Região de Saúde com maior desempenho no indicador. Na Tabela 1 estão descritos os valores utilizados para as variáveis valor mínimo e valor máximo nos 3 parâmetros.

$\mathrm{O}$ intuito de utilizar três parâmetros é verificar as diferenças e semelhanças entre os resultados e evitar o viés do parâmetro.

A próxima etapa é o cálculo do índice do Indicador Composto de Avaliação da Vigilância em Saúde (ICAVES), que consiste na média dos 11 índices parciais de cada Região de Saúde. Desta forma, cada uma das 30 Regiões de Saúde possui um único índice.
O cálculo dos índices parciais e do ICAVES foi realizado para os três parâmetros definidos para a variável valor máximo: (1) Meta recomendada pelo Ministério da Saúde para pactuação do indicador no ano de 2016, (2) Meta pactuada na CIB/ RS para o indicador no ano de 2016 e (3) média da Região de Saúde com maior desempenho no indicador. Assim, o cálculo do ICAVES estadual foi realizado através da média dos índices das 30 Regiões de Saúde.

\section{Resultados}

O Rio Grande do Sul está dividido em 30 Regiões de Saúde compreendidas em 7 Macrorregiões de Saúde.

As Regiões de Saúde 19 (Região do Botucaraí) e 20 (Rota da Produção) apresentaram os menores ICAVES nos três Parâmetros utilizados 
Tabela 1. Valores estabelecidos para as variáveis: valor mínimo e valor.

\begin{tabular}{|c|c|c|c|c|}
\hline \multirow[b]{2}{*}{ Indicador } & \multirow{2}{*}{$\begin{array}{c}\text { Valor Mínimo } \\
\text { Menor } \\
\text { Desempenho }\end{array}$} & \multicolumn{3}{|c|}{ Valor Máximo } \\
\hline & & $\begin{array}{c}\text { Maior } \\
\text { Desempenho }\end{array}$ & $\begin{array}{l}\text { Meta } \\
\text { Ministério } \\
\text { da Saúde }\end{array}$ & $\begin{array}{l}\text { Meta Pactuada } \\
\text { CIB/RS }\end{array}$ \\
\hline $\begin{array}{l}\text { Taxa de mortalidade prematura (de } 30 \text { a } 69 \\
\text { anos) pelo conjunto das quatro principais } \\
\text { doenças crônicas não transmissíveis (DCNT } \\
\text { - doenças do aparelho circulatório, câncer, } \\
\text { diabetes e doenças respiratórias crônicas) }\end{array}$ & 499,55 & 270,44 & 296,51 & 374,16 \\
\hline $\begin{array}{l}\text { Proporção de vacinas do Calendário Básico de } \\
\text { Vacinação da Criança com coberturas vacinais } \\
\text { alcançadas }\end{array}$ & 22,15 & 60,54 & 75,00 & 50,00 \\
\hline $\begin{array}{l}\text { Proporção de cura de casos novos de } \\
\text { tuberculose pulmonar com confirmação } \\
\text { laboratorial }\end{array}$ & 35,86 & 79,25 & 85,00 & 65,00 \\
\hline $\begin{array}{l}\text { Proporção de exame anti-HIV realizados entre } \\
\text { os casos novos de tuberculose }\end{array}$ & 47,63 & 89,93 & 100,00 & 86,90 \\
\hline $\begin{array}{l}\text { Proporção de municípios com casos de } \\
\text { doenças ou agravos relacionados ao trabalho* } \\
\text { notificados }\end{array}$ & 11,85 & 93,33 & 83,00 & 70,00 \\
\hline $\begin{array}{l}\text { Proporção de cura dos casos novos de } \\
\text { hanseníase diagnosticados nos anos das } \\
\text { coortes }\end{array}$ & 45,83 & 100,00 & 88,00 & 88,00 \\
\hline $\begin{array}{l}\text { Proporção de contatos examinados de casos } \\
\text { novos de hanseníase }\end{array}$ & 23,77 & 100,00 & 80,00 & 84,00 \\
\hline Número absoluto de óbitos por dengue & 0,14 & 0,00 & 0,00 & 0,00 \\
\hline $\begin{array}{l}\text { Proporção de imóveis visitados em, pelo } \\
\text { menos, quatro ciclos de visitas domiciliares } \\
\text { para controle da dengue }\end{array}$ & 0,00 & 59,00 & 80,00 & 45,00 \\
\hline $\begin{array}{l}\text { Proporção de análises realizadas em amostras } \\
\text { de água para consumo humano quanto aos } \\
\text { parâmetros coliformes totais, cloro residual } \\
\text { livre e turbidez }\end{array}$ & 29,99 & 110,47 & 100,00 & 80,00 \\
\hline $\begin{array}{l}\text { Percentual de municípios que realizam no } \\
\text { mínimo seis grupos de ações de Vigilância } \\
\text { Sanitária, consideradas necessárias a todos os } \\
\text { municípios }\end{array}$ & 7,69 & 62,50 & 45,00 & 45,00 \\
\hline
\end{tabular}

Nota: Algumas questões observadas ao longo do desenvolvimento do estudo merecem ser descritas:

1. O parâmetro nacional de referência para pactuação das meta do indicador: Taxa de mortalidade prematura (de 30 a 69 anos) pelo conjunto das quatro principais doenças crônicas não transmissíveis (DCNT - doenças do aparelho circulatório, câncer, diabetes e doenças respiratórias crônicas) é a redução de $2 \%$ em relação ao ano anterior. Por essa razão, foi utilizado como valor máximo, no Parâmetro (1), a taxa recomendada para as Américas pela Organização Mundial da Saúde para o ano de 2019 (280/100.000 hab.), com a aplicação da redução de $2 \%$ ao ano para estimar o valor para o ano de $2016(296,51 / 100.000 \text { hab })^{13}$.

2. Para calcular a média da série histórica do indicador - Proporção de imóveis visitados em, pelo menos, quatro ciclos de visitas domiciliares para controle da dengue - foram utilizados somente os anos em que a Região de Saúde foi considerada infestada. Três Regiões de Saúde - R 24 - Campos de Cima da Serra, R 29 - Vales e Montanhas e R 30 - Vale da Luz - não foram consideradas áreas infestadas em nenhum ano. Para essas Regiões de Saúde tal indicador não foi considerado no cálculo do ICAVES, o qual foi calculado utilizando a média de 10 indicadores ${ }^{3}$.

no cálculo: (1) Parâmetro Nacional, (2) Pactuação na CIB/RS e (3) melhor média das 30 séries históricas calculadas para cada indicador. Estas duas Regiões de Saúde estão localizadas na Ma- croregião Norte. A Rota da Produção possui os menores ICAVES: (1) 0,42, (2) 0,51 (3) 0,36. A Região do Botucaraí apresenta os seguintes resultados: (1) 0,44, (2) 0,55 (3) 0,39. 
O ICAVES do Rio Grande do Sul para os $\mathrm{Pa}$ râmetros 1,2 e 3 é, respectivamente , 0,57, 0,72 e 0,52 .

A Figura 1 apresenta o mapa do estado e os resultados do ICAVES.

No Gráfico 1 são apresentados os resultados na forma de gráfico de barras com os valores em ordem crescente de ICAVES sinalizando onde se encontram os primeiro, segundo e terceiro quartis (Q1, Q2 e Q3). As Regiões de Saúde 19 e 20 estão abaixo do primeiro quartil nos três parâmetros, sugerindo que estas devem ser priorizadas para o fortalecimento das ações de Vigilância em Saúde.

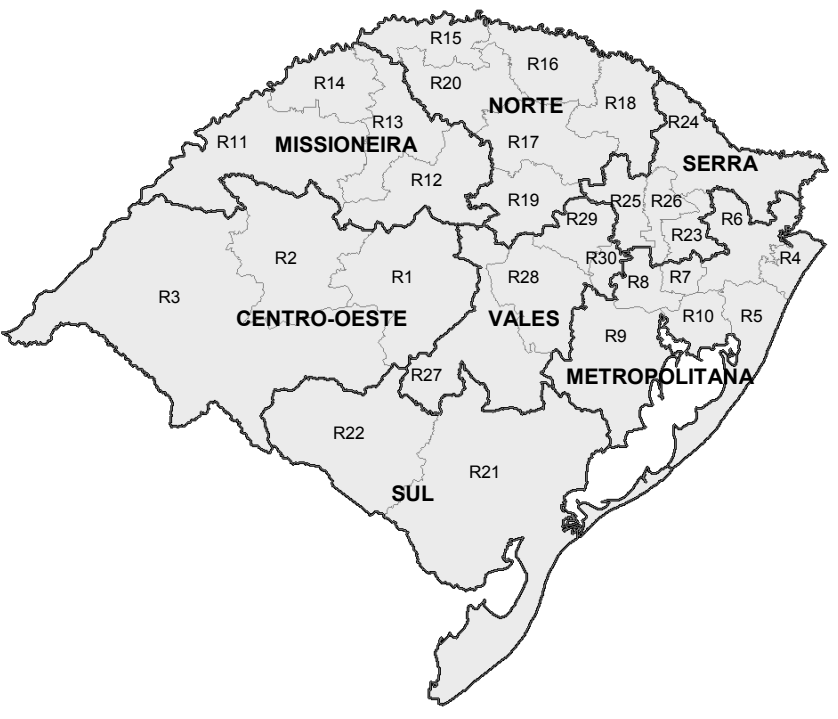

\begin{tabular}{|c|c|c|c|c|}
\hline \multirow{2}{*}{$\begin{array}{c}\text { Macrorregiões } \\
\text { de Saúde }\end{array}$} & \multirow{2}{*}{ Região de Saúde } & \multicolumn{3}{|c|}{ Parâmetros } \\
\hline & & (1) Meta/MS & (2) Meta/CIB/RS & (3) Maior desempenho \\
\hline \multirow[t]{3}{*}{ Centro-Oeste } & R 01 - Verdes Campos & 0,59 & 0,72 & 0,55 \\
\hline & R 02 - Entre-Rios & 0,60 & 0,76 & 0,56 \\
\hline & R 03 - Fronteira Oeste & 0,61 & 0,73 & 0,55 \\
\hline \multirow[t]{6}{*}{ Metropolitana } & R 04 - Belas Praias & 0,53 & 0,66 & 0,47 \\
\hline & R 05 - Bons Ventos & 0,52 & 0,66 & 0,48 \\
\hline & R 06 - Vale do Paranhana Costa da Serra & 0,64 & 0,76 & 0,57 \\
\hline & R 07 - Vale dos Sinos & 0,68 & 0,86 & 0,62 \\
\hline & R 08 - Vale do Caí Metropolitana & 0,56 & 0,69 & 0,51 \\
\hline & R 09 - Carbonífera/Costa Doce & 0,55 & 0,69 & 0,52 \\
\hline \multirow[t]{5}{*}{ Missioneira } & R 10 - CapitalVale do Gravataí & 0,61 & 0,70 & 0,53 \\
\hline & R 11 - Sete Povos das Missões & 0,53 & 0,70 & 0,45 \\
\hline & R 12 - Portal das Missões & 0,55 & 0,68 & 0,52 \\
\hline & R 13 - Região da Diversidade & 0,60 & 0,77 & 0,50 \\
\hline & R 14 - Fronteira Noroeste & 0,66 & 0,86 & 0,66 \\
\hline \multirow[t]{6}{*}{ Norte } & R 15 - Caminho das Águas & 0,57 & 0,69 & 0,51 \\
\hline & R 16 - Alto Uruguai Gaúcho & 0,50 & 0,65 & 0,46 \\
\hline & R 17 - Região do Planalto & 0,54 & 0,69 & 0,41 \\
\hline & R 18 - Região das Araucárias & 0,51 & 0,65 & 0,46 \\
\hline & R 19 - Região do Botucaraí & 0,44 & 0,55 & 0,39 \\
\hline & R 20 - Rota da Produção & 0,42 & 0,51 & 0,36 \\
\hline \multirow[t]{2}{*}{ Sul } & R 21 - Região Sul & 0,45 & 0,55 & 0,41 \\
\hline & R 22 - Pampa & 0,64 & 0,79 & 0,62 \\
\hline \multirow[t]{4}{*}{ Serra } & R 23 - Caxias e Hortências & 0,75 & 0,94 & 0,69 \\
\hline & R 24 - Campos de Cima da Serra & 0,50 & 0,68 & 0,50 \\
\hline & R 25 - Vinhedos e Basalto & 0,75 & 0,98 & 0,70 \\
\hline & R 26 - Uva e Vale & 0,63 & 0,83 & 0,59 \\
\hline \multirow[t]{4}{*}{ Vales } & R 27 - Jacuí Centro & 0,47 & 0,58 & 0,44 \\
\hline & R 28 - Vinte e Oito & 0,61 & 0,76 & 0,57 \\
\hline & R 29 - Vales e Montanhas & 0,67 & 0,84 & 0,64 \\
\hline & R 30 - Vale da Luz & 0,51 & 0,66 & 0,47 \\
\hline
\end{tabular}

Figura 1. Mapa das Macroregiões de Sáude e Regiões de Saúde do Rio Grande do Sul e os valores Indicador Composto da Vigilância em Saúde nos parâmetros (1), (2) e (3). 

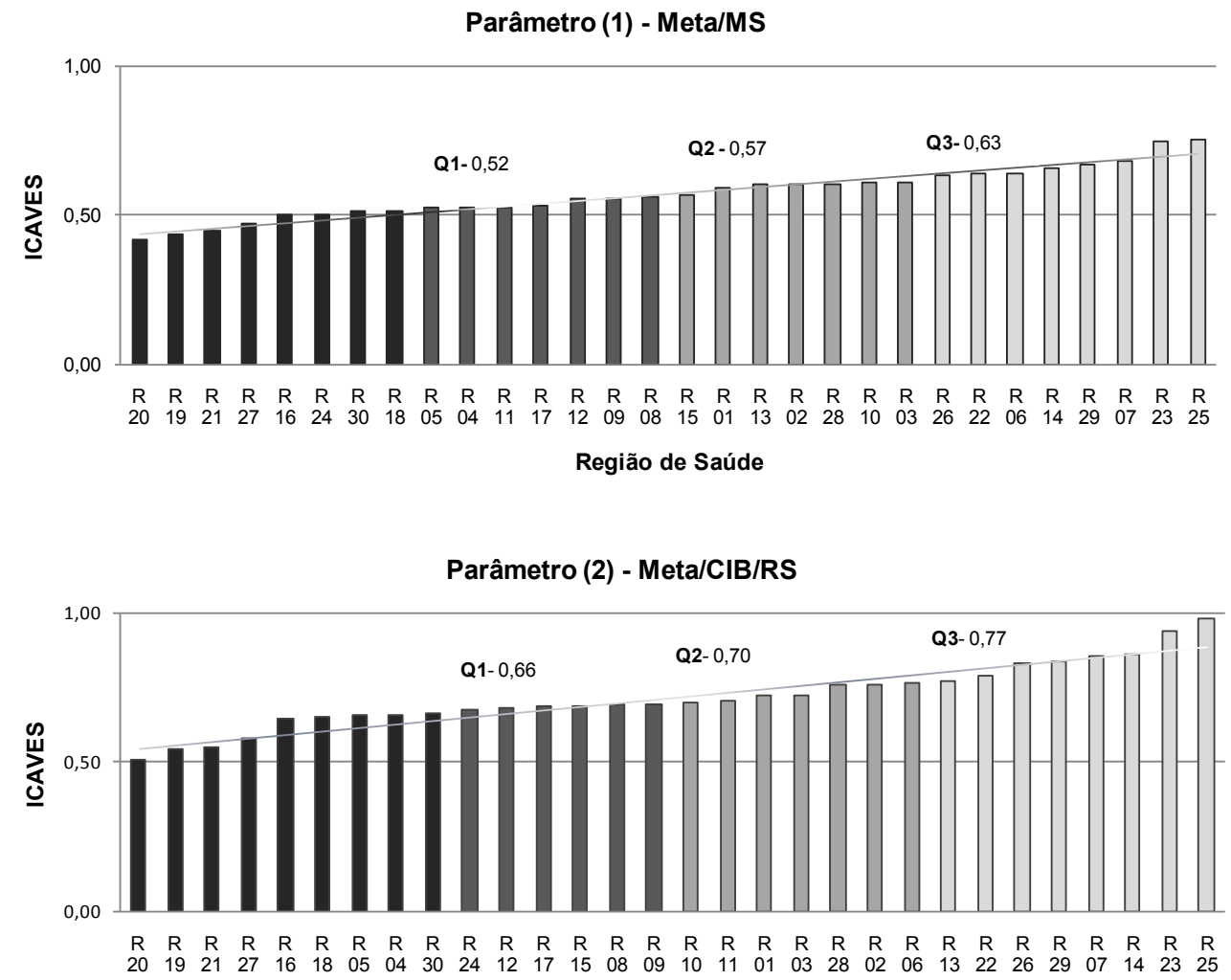

Região de Saúde

Parâmetro (3) - Maior desempenho

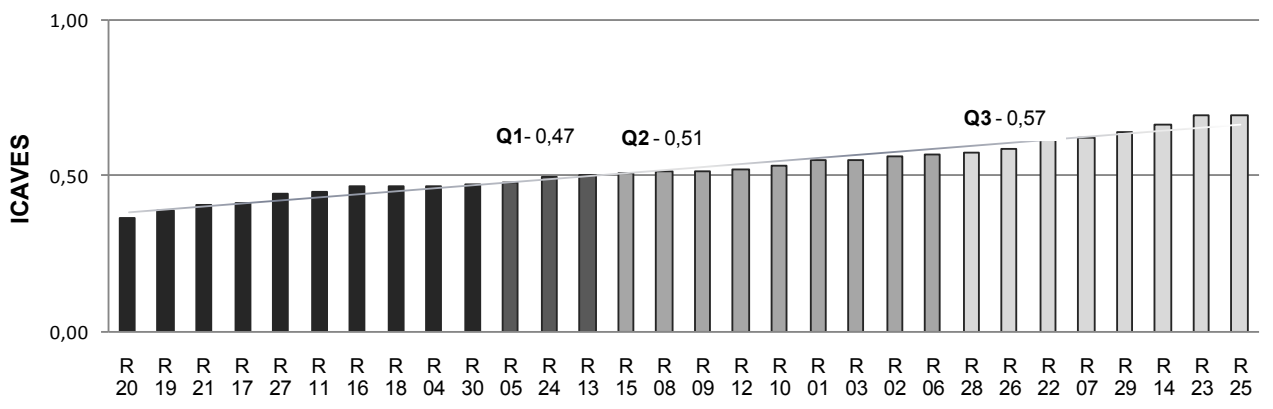

Região de Saúde

Gráfico 1. Indicador Composto da Vigilância em Saúde por desempenho considerando os parâmetros (1), (2) e (3).

Siglas. Q1 - quartil 1; Q2 - quartil 2; Q3 - quartil 3.

\section{Discussão}

O Sistema de Planejamento do SUS considera o Plano de Saúde, em cada esfera, o principal instrumento de gestão, sendo a base para a definição e a implementação de ações e serviços em saú- $\mathrm{de}^{14,15}$. Desta forma, o presente estudo buscou ser compatível com o Plano Estadual de Saúde (PES) 2016-2019 do Rio Grande do Sul, em razão de que sua primeira diretriz (Qualificação da Rede de Atenção à Saúde consolidando a regionalização da saúde) aborda o tema da regionalização e 
inclui o seguinte objetivo: "Fortalecer as ações de âmbito coletivo da Vigilância em Saúde e o gerenciamento de riscos e agravos à saúde" ${ }^{2}$.

Para fortalecer as ações de Vigilância em Saúde é necessário identificar os territórios prioritários, a fim de garantir a equidade. Neste sentido, a avaliação de um conjunto de indicadores produz evidências sobre a situação de saúde e suas tendências, possibilitando identificar as populações e os territórios com maiores necessidades de saúde, estratificar o risco epidemiológico e identificar áreas críticas.

A avaliação dos indicadores da Vigilância em Saúde do Caderno de Diretrizes, Objetivos, Metas e Indicadores 2016 norteou o presente estudo e se mostrou consistente por conter informações apoiadas em dados válidos e confiáveis, assim como indicadores das quatro vigilâncias: ambiental, epidemiológica, sanitária e saúde do Trabalhador (Gráfico 2) ${ }^{4}$.

A Vigilância em Saúde se propõe a trabalhar na lógica de um conjunto articulado e integrado de ações. No entanto, estas ainda se encontram fragmentadas em divisões de vigilância ambiental, epidemiológica, sanitária e saúde do trabalhador, sendo que cada qual atua para seu objetivo de atenção ${ }^{16}$. O Indicador Composto de Avaliação da Vigilância em Saúde proposto permite

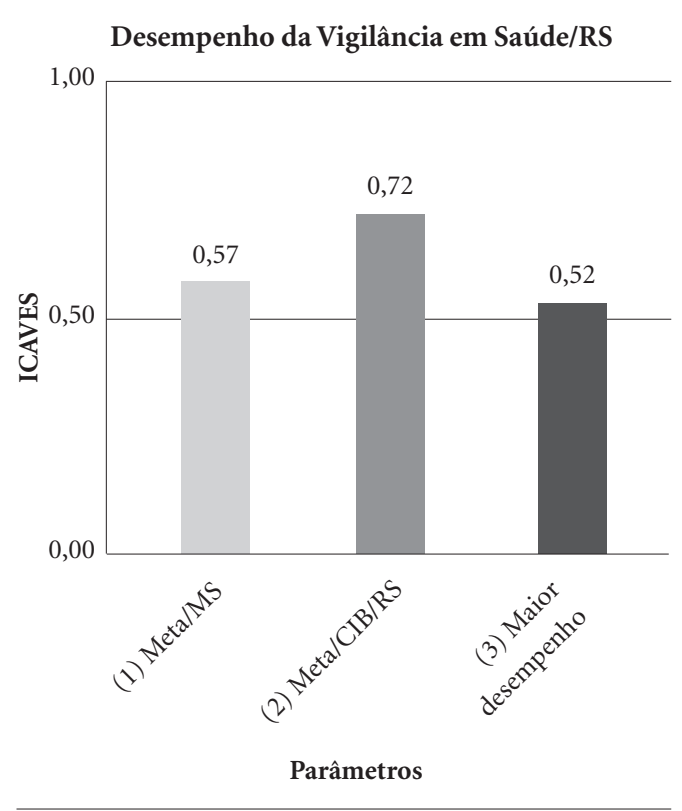

Gráfico 2. Desempenho da Vigilância em Saúde no Rio Grande do Sul nos parâmetros (1), (2) e (3). um diálogo entre as vigilâncias, proporcionando um olhar mais integrado para a gestão ${ }^{16}$.

Analisar o desempenho da Vigilância em Saúde em 30 Regiões de Saúde através da avaliação individual da série histórica de 11 indicadores é uma tarefa que envolve grande número de valores. Neste sentido, o indicador composto é uma alternativa que busca facilitar a avaliação e a comparação entre as 30 Regiões de Saúde. Entretanto, não tem o objetivo de substituir a avaliação individual dos indicadores e sim complementá-la .

Os melhores desempenhos na Vigilância em Saúde são os que apresentam o ICAVES mais próximo do ideal $(1,0)$. A média estadual do ICAVES apresenta valores diferentes nos três parâmetros: (1) Meta recomendada pelo Ministério da Saúde para pactuação do indicador no ano de 2016; (2) Meta pactuada na CIB/RS para o indicador no ano de 2016 e (3) média da Região de Saúde com maior desempenho no indicador; como pode ser visto no Gráfico 2.

Algumas metas pactuadas na CIB/RS são mais baixas que as preconizadas pelo Ministério da Saúde e, por isso, o ICAVES do Parâmetro (2) possui um índice com valor mais alto que o Parâmetro (1). No entanto, o ICAVES do parâmetro (1) e (3) são semelhantes, o que demonstra que a avaliação feita a partir da meta recomendada pelo Ministério da Saúde está coerente com o desempenho real das Regiões de Saúde.

O resultado é um indicativo de que os dados da série histórica dos indicadores em Vigilância em Saúde calculados pelos técnicos Centro Estadual de Vigilância em Saúde do Rio Grande do Sul (CEVS/RS) subsidiaram a pactuação da CIB/RS. Desta forma, se observa o papel do planejamento no setor saúde, nele compreendido o monitoramente e a avaliação de indicadores, que se configura como um relevante mecanismo de gestão conferindo direcionalidade ao processo de tomada de decisão ${ }^{15-17}$.

No Parâmetro (3) as 30 Regiões de Saúde do estado são comparadas a partir do seu desempenho real, nos levando a concluir, sem que haja análise individual dos 11 indicadores, que a amplitude é grande entre a maior e a menor média das séries históricas. Esse resultado aponta que há desigualdade no desempenho da Vigilância em Saúde entre as 30 Regiões de Saúde.

O resultado do cálculo do Indicador Composto de Avaliação da Vigilância em Saúde (ICAVES), nos três Parâmetros utilizados, aponta a Região de Saúde 20 - Rota da Produção como prioritária para o fortalecimento da ações de Vi- 
gilância em Saúde, seguida da Região de Saúde 19 - Botucaraí, ambas localizadas na Macrorregião Norte. Fazendo fronteira com esta, temos a Macrorregião Serra que engloba as Regiões com os melhores resultados: Região de Saúde 25 - Vinhedos e Basalto, seguida da Região de Saúde 23 - Caxias e Hortências (Gráfico 1).

No contexto dos resultados apresentados acima, o apoio matricial pode ser uma estratégia de ação na busca de equidade. As Regiões de Saúde que se destacam por seu desempenho na área de Vigilância em Saúde podem compartilhar seus saberes, competências, responsabilidades e ações com as Regiões de Saúde que apresentam maior dificuldade ${ }^{11,18}$. E de forma complementar, a proximidade geográfica entre as Macrorregiões e/ou Regiões de Saúde pode ser considerada na construção da metodologia do apoio.

O presente trabalho considera que um Indicador Composto da Vigilância em Saúde (ICAVES) possibilita, através de uma metodologia acessível, a síntese de um rol de indicadores, facilitando a análise de um grande quantitativo de dados. Sendo um instrumento robusto, com potencial para contribuir na construção de políticas públicas de saúde com prioridades melhor ajustadas às necessidades da população.

A transversalidade das ações de Vigilância em Saúde na Rede de Atenção à Saúde integra-a com todos os níveis de atenção, tendo como foco principal evitar o adoecimento por meio da detecção, da prevenção e do controle dos fatores determinantes e condicionantes da saúde 3 .

A identificação de áreas prioritárias apoia ações oportunas e induz equidade ${ }^{10}$.

Portanto, é importante destacar a relevância da avaliação dos indicadores de saúde na vigilância das condições de saúde, de forma regionalizada. Visto que propicia intervir em momentos nos quais o risco sanitário pode ser evitado ou minimizado, impactando diretamente na Rede de Atenção à Saúde ${ }^{19}$.

\section{Colaboradores}

RP Mondini trabalhou na concepção, delineamento e redação do artigo; IA Menegolla na concepção, delineamento e revisão crítica; EV Silva na concepção, revisão crítica e redação final.

\section{Agradecimento}

Agradecemos a Secretária Estadual de Saúde do Rio Grande do Sul, em especial ao Centro Estadual de Vigilância em Saúde e ao Grupo de Trabalho de Planejamento, Monitoramento e Avaliação/CEVS, assim como à Escola de Saúde Pública/RS. 


\section{Referências}

1. Estado do Rio Grande do Sul. Secretaria Estadual de Saúde (SES). Resolução no 555/2012. Comissão Intergestores Bipartite 2016. Porto Alegre: SES; 2016.

2. Brasil. Decreto $n^{\circ} 7.508$, de 28 de junho de 2011. Regulamenta a Lei no 8.080, de 19 de setembro de 1990, para dispor sobre a organização do Sistema Único de Saúde - SUS, o planejamento da saúde, a assistência à saúde e a articulação interfederativa, e dá outras providências. Diário Oficial da União 2006; 28 jun.

3. Estado do Rio Grande do Sul. Grupo de Trabalho de Planejamento, Monitoramento e Avaliação da Gestão. Plano Estadual de Saúde: 2016/2019. Porto Alegre: Secretaria Estadual de Saúde; 2016.

4. Brasil. Ministério da Saúde (BR). Caderno de Diretrizes, Objetivos, Metas e Indicadores 2016. Brasília: MS; 2016.

5. Estado do Rio Grande do Sul. Secretaria Estadual de Saúde (SES). Resolução No 33/16. Comissão Intergestores Bipartite 2016. Porto Alegre: SES; 2016.

6. Espaço do Gestor, do Prestador e do Profissional de Saúde. Instrumentos de Gestão e Planejamento. Indicadores Propostos pelo Ministério da Saúde 2016. Secretaria Estadual de Saúde [internet]. 2016 out-nov. [acessado 2016 nov 10]. Disponível em: http://www. saude.rs.gov.br/lista/427/Instrumentos_de_Gest\%C3\%A3o_e_Planejamento

7. Pereira BS, Tomasi E. Instrumentos de apoio à gestão regional de saúde para monitoramento de indicadores de saúde. Epidemiol Serv Saude 2016; 25(2):411-418.

8. Junior JB. Epidemiologia em serviço: uma avaliação de desempenho do Sistema Nacional de Vigilância em Saúde [tese]. Campinas: Universidade Estadual de Campinas; 2004

9. Lei no 8.080 , de 19 de setembro de 1990. Dispõe sobre as condições para a promoção, proteção e recuperação da saúde, a organização e o funcionamento dos serviços correspondentes e dá outras providências. Diário Oficial da União 1990; 19 set.

10. Escorel S. Equidade em Saúde. Diconário da Educação Profissional em Saúde. Fundação Oswaldo Cruz. Escola Politécnica de Saúde Joaquim Venâncio [internet]. 2016 out-nov [acessado 2016 nov 05]. Disponível em: http://www.sites.epsjv.fiocruz.br/dicionario/verbetes/ equsau.html.
11. Righi LB. Apoio matricial e institucional em Saúde: entrevista com Gastão Wagner de Sousa Campos. Comun Saude Educ 2014; 18(1):1145-1150.

12. Gerhardt TE, Silveira DT. Métodos de Pesquisa. Porto Alegre: Editora da UFRGS; 2009.

13. Pan American Health Organization (PAHO). Plan of Action for the Prevention and Control of Noncommunicable Diseases in the Americas 2013-2019. Washington: PAHO; 2014.

14. Brasil. Ministério da Saúde (MS). Sistema de Planejamento do SUS: uma construção coletiva: Instrumentos básicos. Brasília: MS; 2009.

15. Brasil. Ministério da Saúde (MS). Portaria no 3.085, de $1^{\circ}$ de dezembro de 2006. Regulamenta o Sistema de Planejamento do SUS. Diário Oficial da União 2006; $1^{\circ}$ dez.

16. Brasil. Ministério da Saúde (MS). Sistema de Planejamento do SUS: uma construção coletiva: orientaçães gerias para eleaboração de instrumentos de planejamento: Programação Anual de saúde e Relatório Anual de Gestão. Brasília: MS; 2009.

17. Brasil. Ministério da Saúde (MS). Sistema de Planejamento do SUS: uma construção coletiva: monitoramento e avaliação: processo de formulação, conteúdo e uso dos instrumentos do PlanejaSUS. Brasília: MS; 2009.

18. Campos GWS, Domitti AC. Apoio matricial e equipe de referência: uma metodologia para gestão do trabalho interdisciplinar em saúde. Cad Saude Publica 2007; 23(2):399-407.

19. Mendes EV. As redes de atenção à saúde. Brasília: Organização Pan-Americana da Saúde; 2011.

Artigo apresentado em 30/05/2017

Aprovado em 26/06/2017

Versão final apresentada em 19/07/2017 
